\title{
Simulación de anisocoria postraumática
}

\section{Posttraumatic anisocoria simulation}

\section{A. Sibón Olano ${ }^{1}$ MC. Quesada ${ }^{2}$}

P. Martínez García ${ }^{2}$

${ }^{1}$ Médico Forense, Servicio de Patología, Instituto de

Medicina Legal, Cádiz ${ }^{2}$ Médico Forense, Servicio de Clínica Forense, Instituto de Medicina Legal, Cádiz

Correspondencia: Agustín Sibón Olano Instituto de Medicina Legal de Cádiz

C/ Sánchez Barcaiztegui ${ }^{0}$ 3- $2^{0}$ 11071 Cádiz

E-mail: agustin.sibon.ius@ juntadeandalucia.es

Fecha de recepción:

31. OCT. 2013

Fecha de aceptación:

26. MAR 2014

\section{Resumen}

En la práctica forense es relativamente frecuente encontrar individuos que elaboran o exageran síntomas de enfermedades, de manera consciente, con la intención de obtener una ganancia; en otras ocasiones atribuyen malformaciones o patologías previas a un nuevo evento traumático. Por ello es preciso ser minuciosos a la hora de establecer el nexo causal. Se presenta el caso de un individuo que pretende atribuir la secuela de anisocoria traumática a una agresión. El médico forense, con la colaboración de la Policía Científica, pudo demostrar que el origen de la lesión era previo, no pudiéndose establecer el nexo causal.

Palabras clave: Anisocoria traumática. Midriasis traumática. Simulación.

\section{Abstract}

In forensic practice is relatively common to find individuals who produce or exaggerate symptoms of disease, consciously, with the intention of making a profit. Sometimes, malformations or previous pathologies attributed to a new traumatic event. It is therefore necessary to be careful when establishing causation. Today we bring, the case of an individual who seeks to attribute the traumatic aftermath of an attack anisocoria. The Forensic Physician, with the collaboration of the Scientific Police, was able to demonstrate that the origin of the injury was before, not being able to establish a causal link.

Key words: Traumatic anisocoria. Traumatic mydriasis. Simulation.

\section{Caso clínico de una midriasis postraumática atribuida fraudulentamente a una agresión}

\section{Presentación del caso}

Presentamos el caso de un varón de 31 años, peluquero y esteticista de profesión, que refiere como secuela una midriasis postraumática izquierda a consecuencia de lesiones en el curso de una riña, consistentes en una contusión con puño cerrado en el pómulo izquierdo. Entre sus antecedentes de interés sólo figura miopía corregida con lentes. Los diagnósticos son "herida inciso-contusa de $2 \mathrm{~cm}$ en pómulo izquierdo con hematoma periorbitario, erosión nasal derecha, hematoma en cara externa de codo derecho con erosiones y contusiones múltiples, artritis traumática en codo derecho, contusiones y erosiones múltiples en trocánter mayor de cadera izquierda, conjuntivitis y anisocoria postraumática en ojo izquierdo de 1,5 mm en ausencia de anisometropía, trastorno de la acomodación que dificulta su trabajo habitual, disminución del reflejo fotomotor directo y consensual de ojo izquierdo y fotofobia y lagrimeo". El tratamiento recibido consistió en analgésico local, antiinflamatorios orales y antisépticos locales. En las actuaciones judiciales el perjudicado aporta fotografía del pómulo izquierdo del mismo momento de los hechos que evidencia ausencia de herida inciso contusa, a diferencia de los informes privados médicos aportados por el lesionado.

En la demanda judicial se reclama indemnización económica por impedir el ejercicio de su profesión 

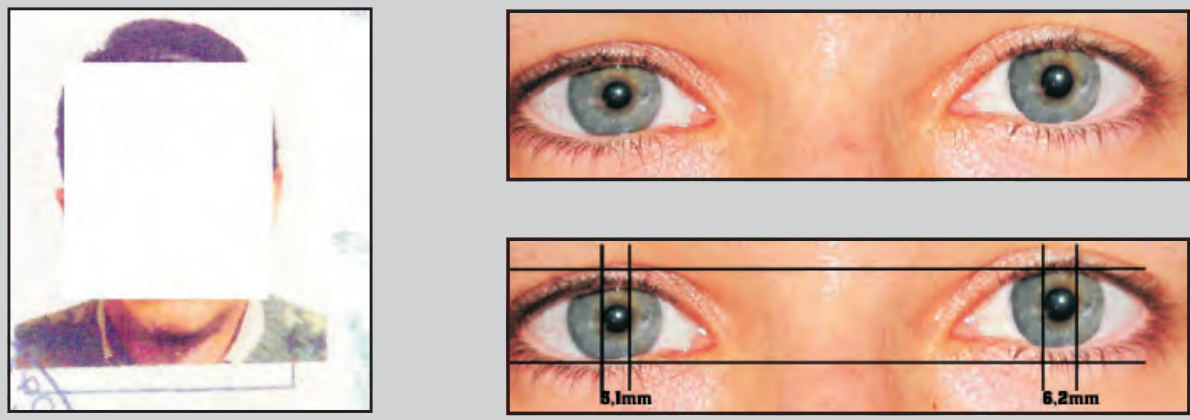

Figura 1. Estudio fisonómico de asimetría ocular en base de datos policial. habitual, así como se pide la calificación como delito con resultado de lesión de órgano principal.

Ante la sospecha de que la midriasis no tuviera un origen postraumático se solicitó un estudio al Cuerpo Nacional de Policía de la fotografía del Documento Nacional de Identidad del lesionado que constaba en sus bases de datos con una data de 4 años previos al relato del suceso, para detectar posibles asimetrías oculares, comprobándose la existencia de "diferencias en el tono muscular aparente de ambos ojos y diferencia en la medida aparente de la pupila de ambos ojos" 4 años antes del suceso.

\section{Discusión}

Los traumatismos oculares pueden clasificarse de múltiples formas ${ }^{1}$ :

- Según el traumatismo: no perforantes, perforantes y otros traumatismos.

- Según el mecanismo lesivo: traumatismo contuso, golpe y contragolpe, herida o solución de continuidad, lesión química, explosiones...

- Según la etiología: accidentes laborales, agresiones físicas, accidentes domésticos, juegos y ocio, y accidentes de tráfico.

- Según la región anatómica afectada: párpados, córnea, conjuntiva, cámara anterior, cristalino, retina y vítreo, coroides, nervio óptico, órbita...

Los delitos violentos son una causa importante de traumatismos oculares, siendo el objeto empleado con mayor frecuencia el puño². Éste ocasiona, normalmente, un traumatismo contuso no perforante que puede producir alteraciones en la cámara anterior, como sería una midriasis asociada generalmente con una parálisis de la acomodación ${ }^{3}$.
La midriasis postraumática es una dilatación o aumento del diámetro pupilar producido por un traumatismo. La fuerza generada durante una contusión ocular lesiona el iris (tanto el músculo del esfínter como el dilatador) y los nervios del iris y del cuerpo ciliar. Así, al contactar bruscamente un objeto romo con la córnea produce una rápida expansión ecuatorial y el desplazamiento enérgico del humor acuoso hacia la periferia de la cámara anterior ${ }^{4}$. Además, según la intensidad puede producirse un vasoespasmo inicial de los vasos uveales anteriores, seguido de una hiperpermeabilidad de los mismos que permite la fuga retrasada de proteínas y fibrina de la cámara anterior, ocasionando una uveítis ${ }^{5}$.

Los pacientes presentan un cuadro de dolor ocular, epifora, fotofobia, molestias con la lectura, visión borrosa y cansancio ocular, y existe correlación entre la sintomatología y el tamaño pupilar. La pupila suele encontrarse en midriasis media, reacciona débilmente a la luz y no existe un defecto pupilar aferente relativo. Aunque los síntomas aparecen con rapidez en los traumatismo graves, en las contusiones leves pueden tardar 3-4 días en presentarse ${ }^{5}$.

El diagnóstico se realiza mediante exploración con lámpara de hendidura, y el pronóstico generalmente es bueno, con resolución en unas pocas semanas. Sólo en un pequeño porcentaje de los casos, cuando existe una lesión importante del esfínter y de los músculos dilatadores, la midriasis traumática es una secuela permanente ${ }^{6}$.

No hay que olvidar el diagnóstico diferencial entre una pupila fija y dilatada, ya que la pupila en midriasis fija es un signo que puede darse en las siguientes situaciones $^{7}$ :

- Pupila tónica de Adie: midriasis unilateral.

- Parálisis del III par craneal.

- Bloqueo farmacológico: es una de las causas más frecuentes. 
- Rotura traumática del esfínter pupilar.

- Glaucoma por cierre angular.

Pero indudablemente, para atribuirle un origen postraumático no basta con la existencia de un traumatismo, sino que deben cumplirse los criterios clásicos de relación de causalidad de $\operatorname{Simonin}^{8}$, que recordamos brevemente:

- Naturaleza del traumatismo: debe ser real, probado, localizado, apropiado y adaptado a las circunstancias. En este caso, analizando la fotografía obrante en autos, no se observa una herida de $2 \mathrm{~cm}$ (como está especificado en el diagnóstico inicial) sino una contusión en la que no se aprecia solución de continuidad.

- Naturaleza de la afección: es necesario que la complicación sea una eventualidad posible y clínicamente admisible.

- Concordancia de asiento: en principio, el traumatismo ha alcanzado de manera directa o indirecta el órgano de la lesión. El lesionado no ha sufrido una contusión directa sobre el globo ocular, sino en el pómulo izquierdo.

- Ligazón anatomoclínica: es necesario que las complicaciones traumáticas estén unidas al traumatismo por una sucesión o cadena de síntomas.

- Condición de tiempo: es el plazo de tiempo entre el traumatismo y la aparición de la sintomatología o de las complicaciones.

- La afectación no existía antes del accidente: esta condición es necesaria para eliminar los hechos de coincidencia. En este caso, gracias al estudio de laboratorio fotográfico se puso de manifiesto que la afectación era anterior a la agresión.
- Exclusión de una causa extraña al accidente: es indispensable que no haya, entre el hecho generador y el hecho consecutivo, interposición de ninguna causa posterior y extraña al accidente.

Por último, siempre hay que tener presente la simulación, que el DSM-IV-TR define como la "producción intencionada de signos o síntomas físicos o psicológicos justificados por incentivos externos (característica que diferencia la simulación de los trastornos facticios)". La sintomatología puede ser inventada, falsificada, autoinflingida, exagerada o exacerbada de un trastorno físico preexistente, o puede ser una combinación o variación de todas las anteriores ${ }^{9}$. Por lo tanto, hay que comprobar la existencia de "falsedad" en los síntomas o signos, y la intención o incentivos que puedan subyacer.

\section{Conclusiones}

La simulación es uno de los problemas medicolegales más frecuentes a los que se enfrenta el médico forense en el ejercicio de sus funciones en la práctica clínica diaria. Así, cuando un lesionado muestra algún síntoma o signo que pueda hacer dudar de la veracidad de sus alegaciones, el profesional debe considerar la posibilidad de que el lesionado mienta o exagere de manera intencional para obtener un beneficio (económico, venganza, eximirse de una obligación legal...). Para detectar estos casos es fundamental un buen reconocimiento del sujeto, un examen de toda la documentación médica, no sólo la aportada por el paciente, y solicitar aquellas pruebas complementarias que se estimen necesarias.

\section{Bibliografía}

1. Moreno Canero F, Fagundez Vargas MA. Traumatismos oculares: aspectos médico-legales. Cuadernos de Medicina Forense. 2002;(29):7-8.

6. Ruiz-Malo R, Domingo ML. Traumatología ocular (I). Contusiones oculares. En: Clement F, editor. Oftalmología. Madrid: Luzán 5. 1994. pp. 68-9.

2. Banta JT. Traumatismos oculares. Editorial Elsevier. 2008. p. 16.

3. Ruiz-Malo R, Domingo ML. Traumatología ocular (I). Contusiones oculares. En: Clement F, editor. Oftalmología. Madrid: Luzán 5.1994. pp. 473-84.

4. Banta JT. Traumatismos oculares. Editorial Elsevier. 2008.pp. 67-8.

5. Banta JT. Traumatismos oculares. Editorial Elsevier. 2008. p. 70.

7. Cursos de Ciencias Básicas y Clínicas. Complemento. Oftalmología básica para estudiantes de medicina y residentes de atención primaria. $5^{\mathrm{a}}$ ed. Editorial Elsevier. pp. 107-8.

8. Simonin C. Medicina legal judicial. Legislación y jurisprudencia españolas. Barcelona: Editorial JIMS. 1922. pp. 284-7.

9. Manual diagnóstico y estadístico de los trastornos mentales IV. Texto Revisado. Barcelona: Editorial Masson. 2002. pp. 575-6, 579. 